\author{
Imma Mautner-Markhof \\ Assistant to the President at Wiener Sängerknaben \\ im Gespräch mit
}

\title{
Gerald Wirth
}

Künstlerischer Leiter und Präsident der Wiener Sängerknaben

\section{Die Wiener Sängerknaben auf dem Weg in die Zukunft}

DOI: https://doi.org/10.53349/sv.2021.i1.a44

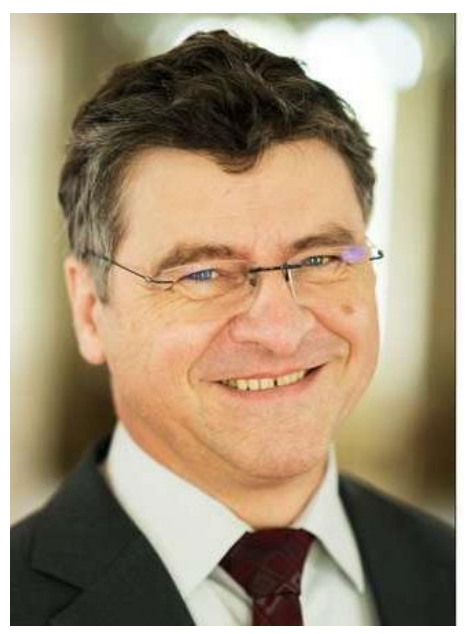

Foto: Lukas Beck

\begin{abstract}
Gerald Wirth erhielt die erste musikalische Ausbildung bei den Wiener Sängerknaben (WSK) und am Bruckner-Konservatorium. Er studierte Oboe, Klavier und Gesang. 2001 wurde er künstlerischer Leiter der WSK, 2013 deren Präsident. 1986-1989 war er Kapellmeister bei den WSK, 1989-1991 Chordirektor am Landestheater Salzburg, 1991-1998 künstlerischer Leiter des Calgary Boys' Choir, Mitbegründer des Vokalensembles Sangita, musikalischer Leiter der Calgary Civic Symphony, Associate Conductor des Calgary Philharmonic Orchestra.

Im Lauf der Jahre entwickelte er seine eigene Unterrichtsmethode - die Wirth Methode. Er hält Workshops an Schulen mit eingeschränktem Zugang zur Musik und für Flüchtlinge, unter anderem in Jordanien und Griechenland. Als Mitgründer von Superar möchte er allen Kindern und Jugendlichen einen qualitätsvollen Zugang zur Musik öffnen.
\end{abstract} quaitatsvollen $u$ uang zurivusik offnen.

\section{Wann fand die erste Berührung mit Musik statt?}

Gerald Wirth: Meine Eltern waren musikinteressiert, wie das am Land üblich war. Ich durfte früh beginnen, ein Instrument zu spielen. Meine Volksschullehrerin hat dann angeregt, dass ich bei den WSK vorsingen sollte. Damit kam ich in die große Stadt. 


\section{Wie wird man Künstlerischer Leiter bei den WSK?}

Mein ehemaliger Kapellmeister war einer der wichtigsten Motivatoren, Musik zu meinem Beruf zu machen. Als Jugendlicher durfte ich schon Kinder- und Jugendchöre leiten, und mit 21 Jahren bot man mir die Chance, einen der Tourneechöre bei den WSK zu übernehmen. Dann wollte ich Neues entdecken und wurde drei Jahre später Chordirektor in Salzburg. Anschließend wurde ich als Künstlerischer Leiter nach Kanada engagiert. Das war ein ideales Feld, um alle Facetten der Tätigkeit eines Künstlerischen Leiters auszuprobieren. Auf Bitte der damaligen Künstlerischen Leiterin der WSK, Agnes Grossmann, ging ich für eine Tournee zurück zu den WSK. Als sie selbst aber während meiner Tournee beschloss, wieder nach Kanada zurückzukehren, erhielt ich die Chance, stellvertretender Künstlerischer Leiter bei den WSK zu werden. Das ließ ich mir nicht entgehen. Anfang 2001 wurde ich zum Künstlerischen Leiter bestellt.

\section{Welche Aufgaben und Herausforderungen hat ein Künstlerischer Leiter?}

In erster Linie geht es darum, die Qualität der WSK und die damit verbundenen Möglichkeiten zu sichern, um den Kindern musikalische Erlebnisse zu bieten. Dazu gehören Nachwuchsarbeit, Zusammenarbeit mit Schulbehörden, die Auswahl musikalischer Mitarbeiter*innen, Unterstützung aller Chorleiter*innen in ihrer Arbeit mit Kindern und bei individuellen Herausforderungen, Programmierung der Tourneen und Konzerte, Auswahl von Projekten im Hinblick auf Probenaufwand und Intensität der musikalischen Tätigkeiten sowie auch die Lösung scheinbar unlösbarer Probleme. Es ist wichtig, in Zeiten intensiver Aktivitäten und bei auftretenden Problemen ein Ruhepol mit offenem Ohr für alle Mitarbeiter*innen, Eltern und Kinder zu sein.

\section{Wie hat sich die Covid-19-Krise auf die WSK ausgewirkt?}

Am 10. März 2020 saß ein Chor bereits im Bus, um zu einer Tournee durch Belgien und die Niederlande aufzubrechen, als uns die Nachricht erreicht hat, dass diese Reise aufgrund von Covid abgesagt werden musste. Das war für uns, wie für alle Kulturschaffenden, das Ende aller Konzerte.

In der Folge musste ein Weg gefunden werden, wie der Probenbetrieb trotz der Pandemie aufrecht erhalten werden konnte, um die Ausbildung der WSK nicht ganz zu vernachlässigen. Für die Institution stellte auch die finanzielle Situation eine große Problematik dar, da wir einen hohen Prozentsatz unseres Jahresbudgets selbst verdienen. Noch schwerwiegender war es jedoch für jene Knaben, die noch keine Möglichkeit gehabt hatten vor Publikum zu singen, internationale Bühnen weltweit zu bereisen und mit tollen Künstler*innen aufzutreten: denn damit ist jener Teil, der allen am meisten Spaß macht, zur Gänze weggefallen. Durch verschiedene Sicherheitsmaßnahmen - wie z.B. Ankauf von Luftreinigungsgeräten, striktes Abstandhalten usw. - konnte ein Teil der Proben stattfinden. Jetzt hoffen wir, durch den Fortschritt der internationalen Impfstrategien in der nächsten Saison wieder auf Konzertreisen fahren zu können. 
In Österreich hatten wir das Glück, dass die Regierung Kulturvereinen, wie wir einer sind, Unterstützung bietet. Diese gleicht zwar die Verluste keineswegs aus, ermöglicht aber das finanzielle Überleben. Am 20. Mai 2021 stand wieder der erste Tour-Bus vor dem Augartenpalais, um die Kinder zum ersten Konzert nach dem Lockdown zu fahren. Wir haben alles daran gesetzt, für diesen Moment gerüstet zu sein, und sind froh, wieder vor Publikum singen zu dürfen.

\section{Führen die WSK sozialpädagogische Projekte im In- und im Ausland durch?}

Es ist uns ein sehr großes Anliegen, dass jedes Kind Zugang zum aktiven Musizieren erhält. Auch in Österreich gibt es viele Familien, die trotz der guten Versorgung mit öffentlichen Musikschulen diese Möglichkeit nicht finden. Daher haben wir mit der Caritas und dem Wiener Konzerthaus das Projekt Superar gegründet, in dem Kinder, vor allem über Schulen, die Möglichkeit haben, kostenfrei in Chor und Orchester zu musizieren, ohne Vorkenntnisse mitbringen zu müssen. Superar arbeitet in mehreren europäischen Ländern: Schweiz, Slowakei, Liechtenstein, Rumänien, Bulgarien und Bosnien \& Herzegowina. Ähnliche Projekte gibt es auch in anderen Ländern und Kontinenten: Das bekannteste ist El Sistema in Venezuela, mit dem ich seit vielen Jahren zusammenarbeiten darf. Über eine europäische Stiftung wurde ich gebeten, ein Projekt in Jordanien mitaufzubauen, das Kinder aus Flüchtlingsfamilien über Musik stärken und ihre Integration fördern soll. Durch ein weiteres soll in öffentlichen jordanischen Grundschulen Musikunterricht angeboten werden. In diesem Projekt bilden wir Grundschulpädagog*innen ohne musikalische Vorbildung aus, damit sie regelmäßig Musik in ihren Unterricht einbauen können. Gerade diese Verbindung musikpädagogischer und humanitärer Aspekte erfordert die beste Methodik und den besten Unterricht. Es freut uns daher sehr, dass das Bundesministerium für Inneres und der Asyl-, Migrations- und Integrationsfonds gemeinsam ein Projekt der WSK mit der Caritas Jordanien fördern.

\section{Wie wird man Wiener Sängerknabe?}

Jeder ist bei uns willkommen, unabhängig von Religion, kulturellen oder sozialen Hintergründen. Man muss nicht unbedingt viel Erfahrung mit Chorgesang haben. Am wichtigsten sind das Interesse und der Spaß am Singen. Wenn die Familie ihr Kind unterstützt, ist das natürlich von Vorteil. Darüber hinaus können wir alles bieten, was das Kind braucht, um ein guter Sängerknabe zu werden. Zu Beginn sollte man telefonisch oder über unsere Homepage einen Termin zum Vorsingen vereinbaren.

\section{Wie haben sich die WSK in den letzten 20 Jahren entwickelt, und wohin soll die Reise ge- hen?}

Die WSK sind weltweit als Konzertchor auf allen großen Bühnen der Welt bekannt. In den letzten 20 Jahren wurde der Aspekt der Ausbildung für alle Altersgruppen verstärkt. Zusätzlich zur koedukativen Volksschule, die es schon lange gibt, wurde ein Oberstufenrealgymnasium mit Schwerpunkt Vokalmusik, auch für Mädchen, eröffnet. Wir möchten allen, die sich später beruflich musikalisch weiterentwickeln wollen, eine gute Vorbereitung und viele küns- 
tlerische Möglichkeiten bieten. Unser Mädchenchor steht allen Mädchen ab 10 Jahren offen. Für Kinder im Vorschulalter gibt es musikalische Früherziehung in der Spielwerkstatt. Das Repertoire hat sich vor allem im Bereich der internationalen Musik aus verschiedensten Kulturen erweitert. Durch den Konzertsaal MuTh können wir das Genre Kinderoper professionell umsetzen und vermehrt Projekte mit Schulkindern durchführen. Das MuTh bietet uns auch die Möglichkeit, künstlerische Projekte im eigenen Konzertsaal zu realisieren, die für kommerzielle Veranstalter vielleicht schwierig wären, aber von großem pädagogischen und künstlerischen Wert sind.Die WSK werden den pädagogischen Aspekt in den nächsten Jahren weiter verstärken, über den traditionellen Knabenchor hinaus, der aber immer der zentrale Punkt bleiben wird. Es ist uns wichtig, allen Kindern höchste musikalische und akademische Qualität zu bieten.

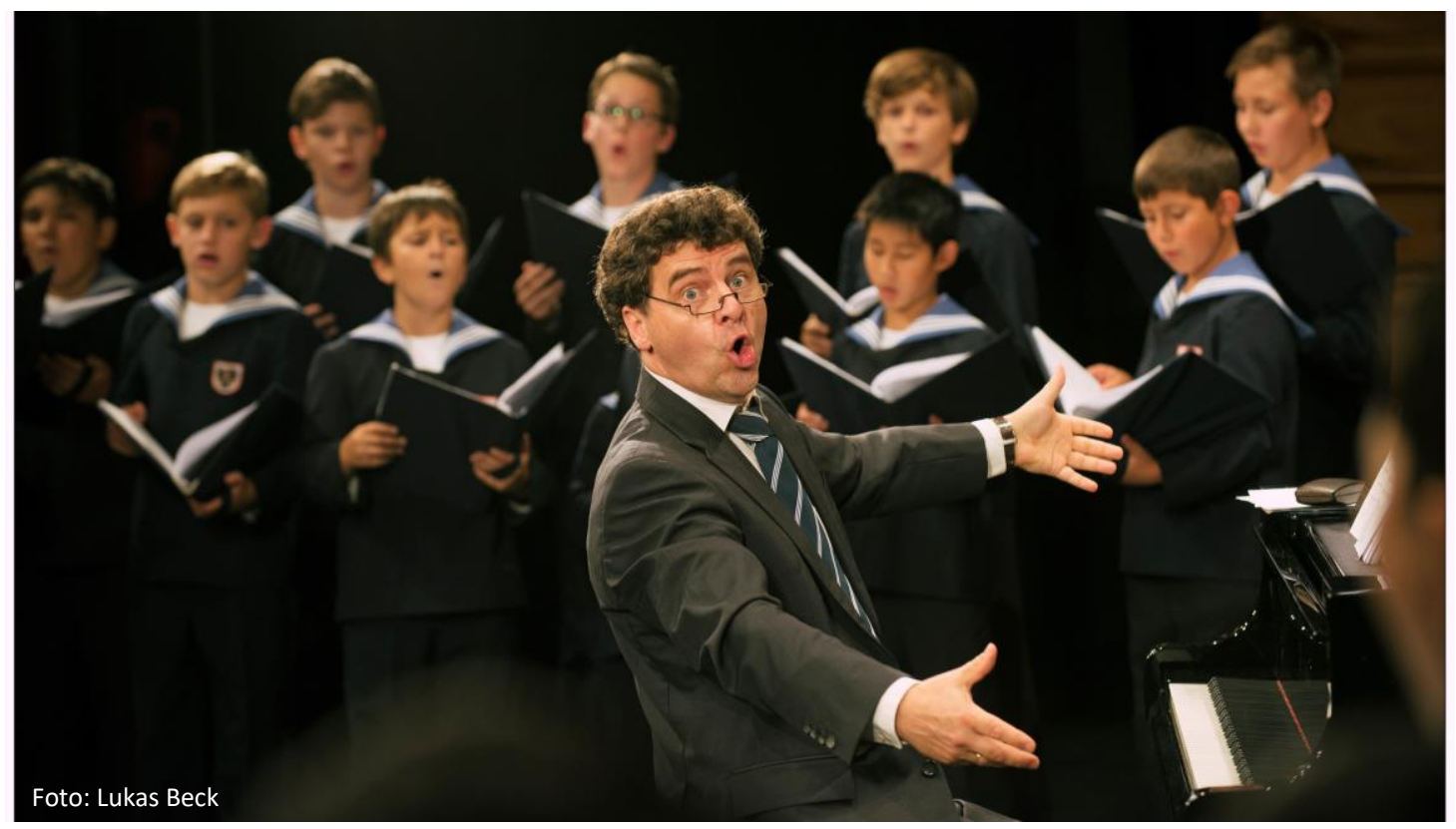

Welche Aspekte der Musikpädagogik können auch für Pädagog*innen ohne Musikerfahrung hilfreich sein?

In unserer Forschungsarbeit und in unserem Training für Musikpädagog*innen setzen wir uns im höchsten Maß damit auseinander, wie wir die aktive Aufmerksamkeit der Kinder erlangen und sie möglichst lange erhalten können. Viele dieser Aspekte sind sicher auch für den Unterricht in anderen Wissensgebieten hilfreich.

\section{Worin besteht diese Forschungsarbeit?}

Mit Hilfe der Familie Pühringer, die uns auch den Konzertsaal ermöglicht hat, konnten wir uns in Zusammenarbeit mit der Athabasca University (CAN) der Forschung widmen, wie unsere Erfahrungen in Bezug auf methodische Aspekte im Musikunterricht am besten digitalisiert und damit einfacher weitergegeben werden können. Mit Hilfe von Psycholog*innen und Spezialist*innen der Lehr- und Lernanalytik haben wir diese Aspekte definiert und in ein digi- 
tales Analysetool eingearbeitet. Diese Software, bei der mit Hilfe von Video- und Tonaufnahmen der Unterricht nach unserer Definition analysiert wird, unterstützt Pädagog*innen in ihrer Selbstreflexion und bietet in Zusammenhang mit unserer E-Learning-Plattform die Möglichkeit, konkrete Lösungsvorschläge und Übungsansätze für diverse Schwierigkeitsgrade im Musikunterricht zu finden. Der Austausch mit pädagogischen Institutionen ist uns sehr wichtig, und daher freuen wir uns besonders über die Zusammenarbeit mit Professor Hubert Gruber, die durch Rektor Erwin Rauscher initiiert worden ist und von Professorin Christine Schörg unterstützt wird. Ich bin überzeugt, dass wir vom reichen Erfahrungsschatz Hubert Grubers im Sinn der von uns unterstützten Projekte profitieren werden und unsererseits einen Beitrag für die Praxisforschungsarbeit der PH NÖ, besonders im Bereich Lesson Study, leisten können. Wir alle haben ein großes, gemeinsames Anliegen: Alle Kinder sollen teilhaben am aktiven Musizieren und Singen.

\section{Info}

https://www.wienersaengerknaben.at/

https://muth.at/ 